PREPARED FOR THE U.S. DEPARTMENT OF ENERGY, UNDER CONTRACT DE-AC02-76CH03073

PPPL-3494

PPPL-3494

UC-70

Control of the Electric Field Profile in the Hall Thruster

by

A. Fruchtman, N.J. Fisch, and Y. Raitses

October 2000
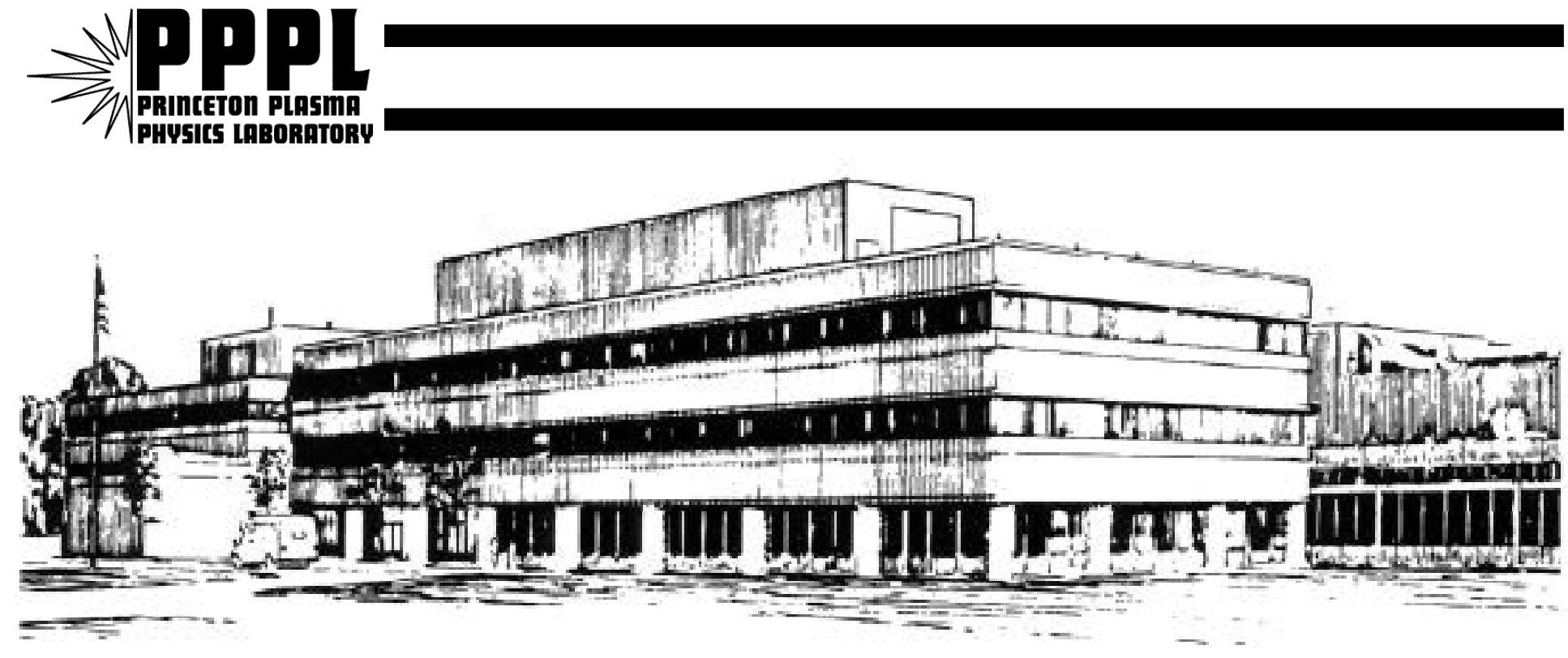

PRINCETON PLASMA PHYSICS LABORATORY PRINCETON UNIVERSITY, PRINCETON, NEW JERSEY 


\section{PPPL Reports Disclaimer}

This report was prepared as an account of work sponsored by an agency of the United States Government. Neither the United States Government nor any agency thereof, nor any of their employees, makes any warranty, express or implied, or assumes any legal liability or responsibility for the accuracy, completeness, or usefulness of any information, apparatus, product, or process disclosed, or represents that its use would not infringe privately owned rights. Reference herein to any specific commercial product, process, or service by trade name, trademark, manufacturer, or otherwise, does not necessarily constitute or imply its endorsement, recommendation, or favoring by the United States Government or any agency thereof. The views and opinions of authors expressed herein do not necessarily state or reflect those of the United States Government or any agency thereof.

\section{Availability}

This report is posted on the U.S. Department of Energy's Princeton Plasma Physics Laboratory Publications and Reports web site in Calendar Year 2000. The home page for PPPL Reports and Publications is: http://www.pppl.gov/pub_report/

DOE and DOE Contractors can obtain copies of this report from:

U.S. Department of Energy

Office of Scientific and Technical Information

DOE Technical Information Services (DTIS)

P.O. Box 62

Oak Ridge, TN 37831

Telephone: (865) 576-8401

Fax: (865) 576-5728

Email: reports@adonis.osti.gov

This report is available to the general public from:

National Technical Information Service

U.S. Department of Commerce

5285 Port Royal Road

Springfield, VA 22161

Telephone: $1-800-553-6847$ or

(703) $605-6000$

Fax: (703) 321-8547

Internet: http://www.ntis.gov/ordering.htm 


\title{
Control of the electric field profile in the Hall thruster
}

\author{
A. Fruchtman \\ Holon Academic Institute of Technology, 52 Golomb St., P.O. Box 305, \\ Holon 58102, ISRAEL \\ N. J. Fisch and Y. Raitses \\ Princeton Plasma Physics Laboratory, Princeton University, \\ P.O. Box 451, Princeton, NJ 08543
}

(October 3, 2000)

Control of the electric field profile in the Hall thruster through the positioning of an additional electrode along the channel is shown theoretically to enhance the efficiency. The reduction of the potential drop near the anode by use of the additional electrode increases the plasma density there, through the increase of the electron and ion transit times, causing the ionization in the vicinity of the anode to increase. The resulting separation of the ionization and acceleration regions increases the propellant and energy utilizations. An abrupt sonic transition is forced to occur at the axial location of the additional electrode, accompanied by the generation of a large (theoretically infinite) electric field. This ability to generate a large electric field at a specific location along the channel, in addition to the ability to specify the electric potential there, allows us further control of the electric field profile in the thruster. In particular, when the electron temperature is high, a large abrupt voltage drop is induced at the vicinity of the additional electrode, a voltage drop that can comprise a significant part of the applied voltage.

PACS numbers: 52.75.Di 


\section{INTRODUCTION}

Electric propulsion for space vehicles utilizes electric and magnetic fields to accelerate a propellant to a much higher velocity than chemical propulsion does, and, as a result, the required propellant mass is reduced. Among electric propulsion devices Hall thrusters offer much higher thrust density than conventional ion thrusters. The Hall thruster accelerates a quasi-neutral plasma, and therefore is not subject to a space-charge limit on the current. An applied radial magnetic field (see Fig. 1) impedes the axial electron motion towards the anode. The impeded electrons can then more effectively ionize the propellant atoms and support a significant axial electric field with equipotentials along the magnetic field lines. The axial electric field accelerates the ions from the anode towards the channel exhaust, in a direction that is opposite to the axial direction of the electrons.

Since the original ideas were introduced [1] - [5], Hall thrusters have enjoyed both experimental and theoretical progress [1] - [27]. Hall thrusters now perform with efficiencies of more than $50 \%$ in the important range of specific impulses of $1500-2500$ seconds. Despite this progress, there is a substantial interest in further improving the thruster performance. This performance is strongly affected by the electric field distribution in the thruster. In a simple Hall thruster, the electric field axial profile is strongly coupled to the magnetic field configuration. Decoupling these profiles might in fact permit improvements in both the thruster efficiency and the plume divergence. In the present paper we demonstrate theoretically means for separately controlling the electric field profile. This control would be gained by the addition of an absorbing electrode between the cathode and the anode and the generation of an abrupt sonic transition inside the channel. We show theoretically how the control of the electric field distribution enables us to increase the thruster efficiency. The effect on the plume divergence will be examined in the future. 
Segmented side electrodes in Hall thrusters have been shown experimentally capable of either supplying or absorbing neutralizing electrons [26]. An additional emitting electrode in the acceleration zone can enhance the current utilization and thus the total efficiency, but the efficiency enhancement is shown theoretically to be very limited[27]. Positioning an additional absorbing electrode in the ionization region, however, can have a greater effect [19]. Here, we show that the increase in the efficiency in this case can be substantial.

We will show that the use of an electrode in the ionization region operates roughly as follows: The reduction of the potential drop near the anode by use of the additional electrode there increases the plasma density there, through the increase of the electron and ion transit times, causing the ionization in the vicinity of the anode to increase. The resulting separation of the ionization and acceleration regions increases the propellant and energy utilizations.

An abrupt sonic transition is forced to occur at the axial location of the additional electrode, accompanied by the generation of a large (theoretically infinite) electric field. This ability to generate a large electric field at a specific location along the channel, in addition to the ability to specify the electric potential there, allows us further control of the electric field profile in the thruster. When the electron temperature is high, an abrupt voltage drop is induced at the vicinity of the additional electrode, large enough to comprise a significant part of the applied voltage.

The idea of deliberately generating an abrupt sonic transition at a specified location along the channel has recently been developed theoretically $[12,14,18,19]$. As in other physical systems [28] - [34], conditions exist in the Hall thruster that guarantee smooth flow through the sonic transition. However, if the sonic transition occurs at the location of an abrupt change of one of the channel features, such as at the boundary of different wall materials with different secondary electron emission coefficients [18], then the sonic transition itself is abrupt. Alternatively, an absorbing electrode can be located at the sonic transition [19], an approach that we pursue further here.

In Sec. II we present the model. In Sec. III we discuss in more detail the nature of the sonic transition in the Hall thruster and the conditions for the formation of an abrupt sonic 
transition. In Sec. IV we demonstrate through several examples the increase in efficiency that results from the addition of an absorbing electrode. We also discuss in Sec. IV the reasons for this efficiency enhancement. We conclude in Sec. V.

\section{THE MODEL}

Hall thrusters are typically coaxial (see Fig. 1), and their configuration is approximately azimuthally symmetric. Because of the low collisionality of the electrons, the magnetic field lines are assumed equipotential. The applied magnetic field is mostly radial, and therefore planes perpendicular to the axial direction are equipotential. The potential inside the channel at such a plane that intersects an electrode is the electrode potential. We model the Hall thruster by an approximate one-dimensional model, in which all quantities vary along the axial direction only, which we denote as the $x$ direction.

The thrust, the specific impulse and the efficiency are the main performance parameters of the thruster. The thrust is defined as

$$
T \equiv \frac{I_{i}(x=L)}{e} m_{i} v_{i}(x=L)
$$

where $e, m_{i}, v_{i}$ and $I_{i}$ are the ion charge, mass, velocity and current. Here $x=0$ at the anode and the length of the channel is $L$. For the calculation of the thrust the values of the ion velocity and current are taken at the thruster exit. The specific impulse is

$$
I_{s p} \equiv \frac{T}{\dot{m} g},
$$

where $\dot{m}$ is the mass flow rate and $g$ is the free-fall acceleration. The thruster efficiency is usually characterized separately by the propellant utilization,

$$
\eta_{m} \equiv \frac{I_{i}(x=L) m_{i}}{e \dot{m}}
$$

the current utilization,

$$
\eta_{C} \equiv \frac{I_{i}(x=L) \phi_{A}}{P},
$$


and the energy utilization,

$$
\eta_{E} \equiv\left[\frac{v_{i}(x=L)}{v_{0}}\right]^{2}
$$

The total efficiency is the product of these three efficiencies

$$
\eta_{T} \equiv \eta_{m} \eta_{C} \eta_{E}=\frac{T^{2}}{2 \dot{m} P}
$$

Here the total dissipated power is $P=-\int_{0}^{L} d x I_{D} d \phi / d x$, where $I_{D}$ is the discharge current, $\phi$ is the electric potential, $\phi_{A}$ is the voltage applied between the cathode and the anode and $v_{0} \equiv\left(2 e \phi_{A} / m_{i}\right)^{1 / 2}$. In the case that $I_{D}$ is constant along the channel $\eta_{C}$ is reduced to $I_{i}(x=L) / I_{D}$

We employ a simple set of fluid equations for the description of the flow. The ion flow is described by the continuity equation

$$
\frac{d j_{i}}{d x}+j_{i} \frac{d \ln A}{d x}=e\left(S-\frac{W d}{A}\right)
$$

and by the momentum equation

$$
m_{i} v_{i} \frac{d v_{i}}{d x}+\frac{m_{i} S v_{i}}{n_{i}}=-e \frac{d \phi}{d x}
$$

Here $n_{i}$ and $j_{i}\left(\equiv I_{i} / A\right)$ are the ion density and current density, $A$ and $d$ are the cross section of the thruster channel and the wall area per unit channel length, $S$ is the source function due to ionization and $W$ is the rate of losses due to recombination at the walls. The ionization acts as a drag force in the momentum equation. We assume though that ions are lost to the walls at a rate that is independent of their $x$ velocity, so that there is no drag due to recombination.

The ion dynamics is governed by the ion momentum equation, where the ions are assumed collisionless and unmagnetized. Since ions are born through ionization along the thruster, the ion pressure is not negligible, but, since the ions are almost collisionless, there is no simple equation of state that relates their pressure to the lower moments. As in our previous papers $[12,18]$, we make the simplifying approximation of neglecting the ion pressure, However, 
although we treat the ions as a cold fluid, we do retain the ion production term through ionization, which, as stated above, appears as an effective drag term.

The electron dynamics is governed by the electron momentum equation

$$
0=-\frac{\left(j_{T}-j_{i}\right) e v_{i}}{j_{i} \mu}-e \frac{d \phi}{d x}+T_{e}\left(\frac{1}{j_{i}} \frac{d j_{i}}{d x}-\frac{1}{v_{i}} \frac{d v_{i}}{d x}\right)
$$

Here

$$
\mu \equiv \frac{e \nu}{m \omega_{c}^{2}}
$$

is the mobility of the electrons across the magnetic field, where $m, \omega_{c}$ and $\nu$ are the electron mass, cyclotron frequency and collision frequency. In writing Eq. (5) we used the assumption that $\nu \ll \omega_{c}$. Also, $j_{T}\left(\equiv I_{d} / A\right)$ is the total current density (so that the electron current density is $j_{e}=j_{T}-j_{i}$ ), and $T_{e}$ is the electron temperature. For the electrons we have included previously an energy equation in our model and showed how one can impose a large temperature gradient along the thruster [14]. For simplicity we will not address the (admittedly important) evolution of the electron temperature here, and we assume that $T_{e}$ is constant along the thruster.

In Eq. (9) the the electron density $n_{e}$ is assumed to be equal to the ion density due to (the assumed) quasi neutrality. Therefore

$$
n_{e}=n_{i}=\frac{j_{i}}{e v_{i}}
$$

We now combine equations (7) - (9) to derive an equation for $v_{i}$ :

$$
\left(v_{i}^{2}-c_{s}^{2}\right) \frac{d v_{i}}{d x}=\frac{e v_{i}^{2}}{m_{i} \mu}\left(\frac{j_{T}-j_{i}}{j_{i}}\right)-\frac{e S v_{i}^{3}}{j_{i}}-\frac{e v_{i} c_{s}^{2}}{j_{i}}\left(S-\frac{W d}{A}\right)+c_{s}^{2} v_{i} \frac{d \ln A}{d x} .
$$

This form of the equation exhibits the singularity at the sonic transition plane. The last term on the right hand side (RHS) of the equation expresses the effect of a varying cross section, the effect that enables a smooth sonic transition at the neck of a Laval nozzle [28].

The boundary conditions are zero ion velocity and current at the anode and a specified voltage between the anode and the catode. These are 


$$
\begin{gathered}
j_{i}(x=0)=0, \quad v_{i}(x=0)=0, \\
\phi(x=0)-\phi(x=L)=\phi_{A} .
\end{gathered}
$$

These boundary conditions for the ion current and velocity at the anode mean that a monotonically decreasing potential from the anode towards the cathode is assumed and the possibility of a backwards ion flow towards the anode is excluded. An analysis of the Hall thruster with such a backwards ion flow has been done recently [15]. When an electrode is added, an additional boundary condition is the voltage between the additional electrode and either the cathode or the anode.

Let us assume that the source function due to ionization is

$$
S=\beta n_{e} n_{a},
$$

and that the rate of losses due to recombination at the walls per unit area is

$$
W=f n_{e} c_{s} .
$$

Here $\beta \equiv\langle\sigma v\rangle, \sigma$ being the ionization cross section and $v$ the electron velocity, $n_{a}$ is the density of the neutral atoms and $c_{s}$ is the ion acoustic velocity. In the isothermal case we assume here the ion acoustic velocity is $\left.c_{s}=\sqrt{(} T_{e} / m_{i}\right)$. Also, $f$ is a number of order unity that depends on the sheath structure near the wall [11]. The sign $<>$ denotes averaging over the electron distribution function.

We express the neutral density as

$$
n_{a}=\frac{\dot{m}}{m_{i} A v_{a}}-\frac{j_{i}}{e v_{a}} .
$$

Here $\dot{m}$ is the mass flow rate. In writing Eq. (15) we used the fact that the sum of the ion flux and the neutral flux is constant along the thruster. We also assumed that the neutrals move ballistically with a velocity $v_{a}$ while their density varies along the channel due to ionization and due to variations in the cross section along the channel.

Equations (7), (8) and (11) are the governing equations. We use them and Eqs. (13) - (15) and write the equations in dimensionless form. The governing equations for the nondimensional ion current $J \equiv j_{i} m_{i} A / e \dot{m}$, ion velocity $V \equiv v_{i} / v_{0}$ and electric potential $\psi \equiv \phi / \phi_{A}$, 
as functions of the axial normalized location $\xi \equiv x / L$ along the thruster channel, are the ion continuity equation

$$
\frac{d J}{d \xi}=p \frac{J}{V}(1-J-q)-J \frac{d \ln A}{d \xi}
$$

the ion momentum equation

$$
\left(V^{2}-C_{s}^{2}\right) \frac{d V}{d \xi}=\frac{V^{2}}{2 \mu_{N}}\left(\frac{J_{T}-J}{J}\right)-p V^{2}(1-J)-p C_{s}^{2}(1-J-q)+C_{s}^{2} V \frac{d \ln A}{d \xi}
$$

and the equation for the electric potential

$$
\frac{d \psi}{d \xi}=-2 V \frac{d V}{d \xi}-2 p V(1-J)
$$

Also, the dimensionless plasma density is $N \equiv J / V$. The dimensionless parameters that appear in the equations are

$$
p \equiv \frac{L \beta \dot{m}}{v_{a} v_{0} m_{i} A},
$$

that measures the strength of the ionization,

$$
\mu_{N} \equiv \frac{\mu \phi_{A}}{L v_{0}}
$$

the dimensionless electron mobility,

$$
q \equiv \frac{f c_{s} d}{\beta n_{a} A}
$$

the dimensionless loss term, and

$$
C_{s}^{2} \equiv \frac{T_{e}}{2 e \phi_{A}}=\frac{c_{s}^{2}}{v_{0}^{2}}
$$

the square of the dimensionless ion acoustic velocity. To Equations (16)-(18) for $J, V$ and $\psi$ we add the boundary conditions: $J(0)=0, V(0)=0, \psi(0)-\psi(1)=1$.

The performance parameters are now expressed with the help of the dimensionless variables. We write the thrust as

$$
T=J(\xi=1) V(\xi=1) \dot{m} v_{0},
$$


and the specific impulse is given by Eq. (2). We also write the efficiencies with the dimensionless variables. They are

$$
\begin{aligned}
& \eta_{m}=J(\xi=1) \\
& \eta_{C}=-\frac{J(\xi=1)}{\int_{0}^{1} d \xi J_{T} d \psi d \xi}, \\
& \eta_{E}=V^{2}(\xi=1) .
\end{aligned}
$$

In the next section we discuss acceleration to supersonic velocities with an abrupt sonic transition and compare it to the acceleration with a regular smooth transition.

\section{THE SONIC TRANSITION}

The sonic transition in the Hall thruster has similarities to the sonic transition in other flows. The singularity that appears at the sonic transition in the fluid equations that describe such flows, reflects the difference in the nature of acceleration between the subsonic and the supersonic regimes. In the subsonic regime the effect of the pressure is too large, and it is the presence of a $d r a g$ force that is essential for a steady acceleration to occur. In the supersonic regime, on the other hand, the effect of the pressure is not large enough, and an additional accelerating force is necessary. At the sonic transition the drag force and the accelerating force should balance. Therefore, the RHS of the momentum equation (as in Eqs. (11) and (17)) should be negative at the subsonic regime, positive at the supersonic regime, and zero at the sonic transition. These relations among the various forces always exist in the smooth acceleration to supersonic velocities. In the Laval nozzle the varying cross section of the channel acts as a force, a drag force when the channel converges in the subsonic regime, and an accelerating force when the channel diverges in the supersonic regime. The sonic transition occurs at the neck of the nozzle, where the cross section of the channel is at its minimum [28]. The role of a converging geometry in the subsonic regime could be played by other drag terms. Such are the ablative and the dissipative terms in ablative discharge capillaries [34], and the sun gravitation in the solar wind [31]. In all these cases the flow 
through the sonic transition is smooth.

The role of the sonic transition in the Hall thruster is only recently being explored $[12,14,15,18-21]$. We have discussed in some detail the above physics issues related to the sonic transition [19], while we analyzed the sonic transition in the Hall thruster configuration. In the Hall thruster a varying cross section is not essential for the acceleration. It is the force due to the magnetic field pressure (expressed as the first term on the RHS of Eqs. (9), (12) and (18)) that accelerates the plasma. However, in the Hall thruster as well it is the presence of a drag force (due to ionization) that is essential for the occurrence of a steady acceleration in the subsonic regime. At the sonic transition the force due to the magnetic field pressure and the drag due to ionization balance.

As in other flows, the acceleration to supersonic velocities in the Hall thruster is usually smooth. The singularity in the fluid equations does not result in the Hall thruster in an abrupt change in the flow, as it does not in other flows. It has been pointed out, probably without noticing the relation to the sonic transition, that in the anode layer (a variation on the Hall thruster in which the electron temperature is relatively high), the fluid equations are singular, and it was suggested that the singularity induces a strong electric field that is localized in a very narrow region $[3,6,24,25]$. We, however, do not expect a strong electric field to arise spontaneously at the sonic transition plane inside the channel due to the singularity in the equations. As was described above, despite this singularity, we expect the flow that is accelerated to supersonic velocities usually to be smooth $[12,14,18,19,21]$.

There are cases in which a sonic transition is followed by a large electric field. Such an abrupt sonic transition is induced by abrupt changes at the channel features. For example, a well known such acceleration exists at a plasma-surface boundary, where a sheath is formed at such a boundary if the Bohm criterion (that the ion flow into the sheath is supersonic) is met $[35,32]$. The acceleration in the supersonic regime in this case occurs in a nonneutral plasma. The quasi-neutral equations yield an infinite electric field, and the singularity is resolved by resorting to Poisson's equation for analyzing the nonneutral plasma. Also, when parameters are adjusted it is possible that the sonic transition occurs at the channel exit. The 
flow is then subsonic inside the channel and supersonic outside the channel, with an abrupt change at the exit $[15,19]$. Being interested in generating an abrupt sonic transition at a specified location inside the channel, we suggest that this can be done by imposing an abrupt change in the thruster parameters at the sonic transition plane along the channel. We have first demonstrated that such an abrupt change could be a change of the secondary emission coefficient of the thruster walls [18], and later we showed that it could be the presence of an additional absorbing electrode [19]. We note that non-neutral regions in which the electric field is large, called double layers, are also known to be formed in a plasma far from its boundary in space and laboratory plasmas [37]. These double layers are not necessarily related to the sonic transition, however, and, if electrostatic, do not exert a net momentum change onto the plasma. In magnetized plasmas, such as in the Hall thruster, the large electric field generated at the sonic transition plane in the way we propose, is followed by a large magnetic field pressure, and, therefore, exerts a large net force on the plasma.

In the next section we show through numerical examples how the addition of an electrode along the thruster may enhance the thruster efficiency. An abrupt sonic transition occurs at the axial location of the additional electrode.

\section{NUMERICAL EXAMPLES}

In this section we compare the performance of a Hall thruster in which an abrupt sonic transition occurs at an additional electrode with the performance of a regular Hall thruster in which the sonic transition is smooth. For both types of Hall thruster we specify the magnetic field profile, the mass flow rate, and the voltage applied between the cathode and the anode. In the Hall thruster with the additional electrode we also specify the position and the electric potential at the additional electrode. We solve for the profiles along the channel of the flow variables (the ion and electron currents and the ion velocity) and of the electric potential, and for the total current along the channel (or equivalently for the electron current

emitted from the cathode). In the Hall thruster with the additional electrode we solve for 
the total currents in the two regions, between the anode and the additional electrode and between the additional electrode and the cathode (or equivalently for both electron current that is emitted from the cathode and electron current that is absorbed at the additional electrode). Once the flow variables are calculated, the efficiencies are also determined.

In the Hall thruster with the additional electrode the sonic transition can occur either in the region between the anode and the additional electrode or in the region between the additional electrode and the cathode. Once the flow parameters and the applied voltage are specified, the location of the sonic transition is determined by specifying the potential at the additional electrode. In all the examples presented here we choose the case that the sonic transition occurs at the additional electrode, so that the flow is subsonic in one region and supersonic in the other region. The total currents are generally different, the current closer to the cathode being larger, thus the additional electrode is absorbing.

The numerical procedure for finding steady flows is somewhat different for the two types of Hall thruster. For the regular Hall thruster we find solutions that are smooth by requiring that the RHS of Eq. (17) be zero at the sonic plane. Note that by looking for smooth solutions and by requiring that the RHS of Eq. (17) vanish at the sonic plane, we essentially specify the value of the total current (or equivalently of the electron current emitted from the cathode). We calculate the finite derivative of the flow velocity there by employing L'Hôpital rule and calculating the ratio of the derivatives of the RHS and of the coefficient in front of the derivative on the left hand side (LHS) of the equation. Then, by expressing the derivative of $J$ by Eq. (16), we find an algebraic second order equation for the derivative of the flow velocity at the sonic plane. With this expression, we also find the finite value of the derivative of the electric potential at the sonic transition plane. The location of the transition plane is found by a shooting method, which assures also that the boundary conditions are satisfied. We integrate the equations from the sonic plane in both directions, towards the cathode and towards the anode. We adjust the location of the sonic transition plane and the values of the ion current and velocity there until the solution satisfies the boundary conditions at the anode and at the cathode. 
Employing this procedure, we have performed a parameter study of the regular Hall thruster (with a smooth sonic transition) [12,21]. As may be expected, the higher is $p$, the higher is the propellant utilization (and to a certain extent the higher is also the energy utilization). The current utilization is increased primarily by the reduction of the electron mobility $\mu_{N}$ along the thruster. The total efficiency generally increases with the increase of $p$ and the decrease of $\mu_{N}$. Steady solutions though exist only above a certain minimal value of $\mu_{N}$, which is different for different values of $p$. These theoretical findings [12,21] coincide with experimental findings that there are regimes of operation in which the flow seems to be steady, while in other regimes the flow is not steady but rather oscillatory and even unstable.

For the Hall thruster with an additional electrode at a specified location along the channel, we employ the following numerical procedure. For specified magnetic field profile, mass flow rate and applied voltage between the anode and the cathode, we vary the potential at the additional electrode until the sonic transition occurs there. In fact, we perform the equivalent procedure of varying the total current in the region between the anode and the additional electrode until the sonic transition occurs at the additional electrode; the potential at the additional electrode being specified when the total current is specified. Now that the flow in the subsonic regime is obtained we turn to the second region, between the additional electrode and the cathode. Taking as boundary conditions the values of the ion current and the ion velocity at the additional electrode and the specified-by-now voltage between the additional electrode and the cathode, we solve for the distribution of the flow variables and of the electric potential and also for the value of the total current in the second region.

The RHS of Eq. (17) is negative in the region near the anode and positive in the region near the cathode. It does not vanish at the sonic transition but its value changes abruptly from negative to positive due to the abrupt change in the total current that is induced by the absorption of part of the current by the additional electrode. Because the RHS of Eq. (17) does not vanish at the sonic transition plane, the electric field and the acceleration are 
very large (theoratically infinite) there.

In all three examples given here the electron mobility is of the form

$$
\frac{1}{\mu_{N}}=\frac{1}{\mu_{0}} \xi
$$

Such a mobility corresponds to the usual magnetic field profile in which the intensity increases from the anode towards the cathode. We can write the normalized mobility as

$$
\frac{1}{\mu_{0}}=1.76 \cdot 10^{9} \frac{B^{2} v_{0}}{\nu \phi_{A}}
$$

where

$$
v_{0}=1.38 \cdot 10^{4}\left(\frac{\phi_{A}}{A_{N}}\right)^{1 / 2} \frac{m}{s}
$$

In Eqs. (26) and (27) B, the maximal intensity of the magnetic field, is in tesla, $v_{0}$ is in meters per second, $\phi_{A}$ is in volts, $\nu$ is in inverse seconds, and $A_{N}$ is the atomic mass number. For example, when the applied voltage is $300 \mathrm{~V}$, the gas is xenon, the maximal magnetic field intensity is $300 \mathrm{G}$, and $\nu$ is $5.8 \cdot 10^{6} \mathrm{~s}^{-1}, v_{0}$ and $1 / \mu_{0}$ turn out to be $2.1 \cdot 10^{4} \mathrm{~m} / \mathrm{s}$ and 19 . This value of the collision frequency is about seven times larger than the collision frequency expected from binary collisions [38] for a plasma of a density $10^{12} \mathrm{~cm}^{-3}$ and a temperature of $15 \mathrm{eV}$, but about 35 times smaller than the effective collisionality due to near wall resistivity for a distance of $1 \mathrm{~cm}$ between the walls [7].

Let us now estimate the values of $p$ and $q$ for typical parameters of the Hall thruster. For Xenon a typical value of $\beta$ is $3 \cdot 10^{-8} \mathrm{~cm}^{3} \mathrm{~s}^{-1}$. If the thruster cross section and length are $A=20 \mathrm{~cm}^{-2}$ and $L=2 \mathrm{~cm}$, and the gas mass flow rate and velocity are $\dot{m}=2.2 \mathrm{mg} / \mathrm{s}$ and $v_{a}=100 \mathrm{~m} / \mathrm{s}$, the parameter $p$ turns out to be 1.42 . For a neutral density of $5 \cdot 10^{13} \mathrm{~cm}^{-3}$ and a sonic velocity of $3.34 \cdot 10^{5} \mathrm{~cm} / \mathrm{s}$ (corresponding to $15 \mathrm{eV}$ electron temperature) and taking $d / A=2 /\left(r_{o}-r_{i}\right)$ to be $1 \mathrm{~cm}^{-1}$, we find $q$ to equal $0.52 f$.

In the figures we compare the profiles of the ion, electron and total currents, the ion velocity, the plasma density and the electric potential in the regular Hall thruster to those profiles in a Hall thruster with an additional electrode. Three cases are shown, in all of them the addition of the electrode increases the efficiency. 
The reasons for the increase in efficiency are the following. One cause of low efficiency in the Hall thruster, expressed as a low propellant utilization, is that the electron density is too low (due to a too short electron transit time) to enable a full ionization of the propellant. Also, the extension of the acceleration zone into the ionization zone, when occurring in the Hall thruster, results in an inefficiency, expressed as a low energy utilization, since some ions do not acquire the energy that corresponds to the the full voltage drop. With an appropriate biasing, the additional electrode reduces the potential drop near the anode and results in a localization of the main voltage drop closer to the exit. The resulting increase of the electron and ion transit times in the vicinity of the anode increases the plasma density near the anode. As a result of the higher density, the ionization is increased in the vicinity of the anode. The localization of the main voltage drop closer to the exit is therefore used to advantage to increase the plasma density near the anode and to separate the ionization and acceleration regions, thus enhancing both the propellant and energy utilizations. A decrease in the current utilization often follows the addition of an absorbing electrode, since a larger electron current flows in the region nearer to the cathode, where the potential drop is larger. However, the increase in the propellant and energy utilizations, due to the decrease of the potential drop in the ionization region, is often dominant.

Let us examine the three examples. Figure 2 presents a case in which the efficiency is high, because of the high values of $p(=1.42)$ and $1 / \mu_{0}(=19)$ and the lack of wall losses $(q=0)$. The electron temperature is $T_{e} / e \phi_{A}=0.05$. The additional electrode is located at $\xi=0.3$. It is seen that, as suggested above, the positioning of an electrode with a potential only slightly lower than the anode potential makes the ion and electron velocities small in this region. As a result the plasma density for given ion and electron fluxes is higher (as seen in the density profiles) and the rate of ionization is higher. This is expressed both in the higher plasma density, especially in the anode neighborhood, and in the ionization taking place nearer to the anode. The effect of the improved ionization on the propellant utilization is not very dramatic in this case, since the propellant utiliization is very high anyway; it is increased from $\eta_{m}=0.929$ to $\eta_{m}=0.996$. The energy utilization, however, is increased 
significantly, from $\eta_{E}=0.771$ to $\eta_{E}=0.979$. The current utilization decsreases due to the electrode addition from $\eta_{C}=0.927$ to $\eta_{C}=0.867$. However, the substantial increase in the energy utilization, due to the decrease of the potential drop in the ionization region, is dominant. As a result, the total efficiency is enhanced from $\eta_{T}=0.664$ to $\eta_{T}=0.845$.

A substantial increase in the efficiency is also shown in Fig. 3, for a case of low efficiency. Here the parameters $p(=0.75)$ and $1 / \mu_{0}(=3)$ are smaller than in the case shown in Fig. 2, but the wall losses are zero $(q=0)$ and the temperature is $T_{e} / e \phi_{A}=0.05$ as well. Again, the addition of the electrode (here at $\xi=0.4$ ) reduces the current utilization and increases the propellant and the energy utilizations. The current utilization decreases from $\eta_{C}=0.521$ to $\eta_{C}=0.482$. However, here the enhancement of the propellant utilization is substantial, since in the regular Hall thruster the propellant utilization is low, $\eta_{m}=0.707$. The decrease in the potential drop near the anode due to the addition of the electrode increases about four times the electron density, as is seen in the figure. The ionization near the anode increases, and, as a result, the propellant utilization increases significantly, from $\eta_{m}=0.707$ to $\eta_{m}=0.972$, and the energy utilization, from $\eta_{E}=0.763$ to $\eta_{E}=0.969$. The total efficiency increases from $\eta_{T}=0.281$ to $\eta_{T}=0.454$.

Figure 4 presents a case of a relatively high temperature. The remperature is $T_{e} / e \phi_{A}=0.5$, instead of $T_{e} / e \phi_{A}=0.05$ in the cases shown in Figs. 2 and 3 . In the case in Fig. 4 there were wall losses, so that $q=0.1$. In this high temperature case all three efficiencies are increased by the addition of the electrode at $\xi=0.8$. The propellant utilization is increased from $\eta_{m}=0.433$ to $\eta_{m}=0.776$ and the energy utilization from $\eta_{E}=0.471$ to $\eta_{E}=0.761$. The current utilization is also increased in this case, from $\eta_{C}=0.593$ to $\eta_{C}=0.648$. The total efficiency is increased from $\eta_{T}=0.124$ to $\eta_{T}=0.378$.

In this case of high temperature, a large abrupt voltage drop is induced by the sonic transition at the vicinity of the additional electrode, a voltage drop that comprises a significant part of the applied voltage. Nevertheless, the increase in efficiency in this case is mostly a result of the weakening of the electric field in the ionization region, and not of the abrupt potential drop generated near the additional electrode. The use of the abrupt voltage drop, 
demonstrated here, for efficiency enhancement and plume collimation will be examined in the future.

\section{CONCLUSIONS}

In this paper we have demonstrated theoretically a control of the electric field distribution in the Hall thruster that does not rely on a localization of the magnetic field. The control of the electric field is achieved by the addition of an absorbing electrode in the ionization region and by generating an abrupt sonic transition. The location of the maximal potential drop can be controlled by controlling the location of the sonic transition. This additional flexibility relieves us of the necessity of tailoring the magnetic field to control this drop. However, the magnitude of the localized voltage drop that can indeed be formed is significant only when the electron temperature is substantial.

We examined here the consequences of both positioning an absorbing electrode and forcing an abrupt sonic transition at that electrode. We numerically demonstrated through several examples efficiency enhancement in the Hall thruster through modification of the electric field profile by the addition of the absorbing electrode. The abrupt sonic transition did not seem crucial to the efficiency enhancement in these examples. In the future we will continue to further explore the separate roles of the additional electrode and the abrupt sonic transition. We will examine the simpler case of an additional electrode where the sonic transition does not occur at the vicinity of the additional electrode, and the electrode is located either in the subsonic regime or in the supersonic regime. Configurations in which the abrupt sonic transition itself affects the efficiency will be explored. In subsequent studies we will also examine how each of the means described here, the segmented electrodes and

the abrupt sonic transition, affect the plume collimation. Evidence of that has been recently seen experimentally [26].

Some questions related to the approximations in the model should also be addressed, such as the approximation of a constant temperature along the channel. Absorption of a 
substantial part of the electron current by the additional electrode may affect the electron temperature in the ionization region near the anode. The model should be modified to address this issue. Another issue that should be addressed is the uniqueness of the steadystate solutions.

Although the results presented are not yet comprehensive, they clearly demonstrate the ability to control the electric field profile in the Hall thruster, and a way in which this control can be used in certain cases for efficiency enhancement.

\section{ACKNOWLEDGEMENTS}

This research has been partially supported by the United States Air Force Office of Scientific Research and by a Grant No. 9800145 from the United States-Israel Binational Science Foundation (BSF), Jerusalem, Israel. 
[1] P. M. Morozov, in "Physics and Problems of Controlled Fusion" (USSR Academy of Science, Moscow, 1958), Vol. 4, pp 235-257 (in Russian).

[2] R. J. Etherington and M. G. Haines, Phys. Rev. Lett. 14, 1019 (1965).

[3] A. V. Zharinov and Yu. S. Popov, Sov. Phys. Tech. Phys. 12, 208 (1967).

[4] Robert G. Jahn, "Physics of Electric Propulsion" (McGraw-Hill, New York, 1968), Chap. 8.

[5] A. I. Morozov, Yu. V. Esipchuk, G. N. Tilinin, A. V. Trofinov, Yu. A. Sharov, and G. Ya. Shahepkin, Sov. Phys. Tech. Phys. 17, 38 (1972).

[6] H. R. Kaufman, AIAA Journal 23, 78 (1985).

[7] A. I. Bugrova, A. I. Morozov, and V. K. Kharchevnikov, Sov. J. Plasma Phys. 16, 849 (1990).

[8] E. Y. Choueiri, "Characterization of oscillations in closed drift thrusters", AIAA paper 943013, 30th Joint Propulsion Conference, Indianapolis, IN 1994 (American Institute of Aeronautics and astronautics, Washington, DC, 1994).

[9] Y. Raitses, J. Ashkenazy, and M. Guelman, AIAA paper 96-3193, 32th Joint Propulsion Conference, Lake Buena Vista, FL 1996 (American Institute of Aeronautics and astronautics, Washington, DC, 1996).

[10] M. Hirakawa and Y. Arakawa, AIAA paper 96-3195, 32th Joint Propulsion Conference, Lake Buena Vista, FL 1996 (American Institute of Aeronautics and astronautics, Washington, DC, 1996).

[11] J. M. Fife, M. Martinez-Sanchez, and J. Szabo, AIAA paper 97-3052, 33th Joint Propulsion Conference, Seattle, WA 1997 (American Institute of Aeronautics and astronautics, Washington, DC, 1997).

[12] A. Fruchtman, N. J. Fisch, J. Ashkenazy, and Y. Raitses, IEPC paper 97-022, 25th Interna- 
tional Electric Propulsion Conference, Cleveland, OH 1997.

[13] J. Ashkenazy, Y. Raitses, and G. Appelbaum, Phys. Plasmas 5, 2055 (1998).

[14] A. Fruchtman and N. J. Fisch, AIAA paper 98-3500, 34th Joint Propulsion Conference, Cleveland, OH 1998 (American Institute of Aeronautics and astronautics, Washington, DC, 1998).

[15] E. Ahedo and M. Martinez-Sanchez, AIAA paper 98-8788, 34th Joint Propulsion Conference, Cleveland, OH 1998 (American Institute of Aeronautics and astronautics, Washington, DC, 1998).

[16] L. B. King and A. D. Gallimore, Phys. Plasmas 6, 2936 (1999).

[17] W. A. Hargus and M. A. Cappelli, AIAA paper 98-3336, 34th Joint Propulsion Conference, Cleveland, OH 1998 (American Institute of Aeronautics and astronautics, Washington, DC, 1998).

[18] J. Ashkenazy, A. Fruchtman, Y. Raitses, and N. J. Fisch, Plasma Physics and Controlled Fusion 41 (1999) A357.

[19] A. Fruchtman and N. J. Fisch, AIAA paper 99-2142, 35th Joint Propulsion Conference, Los Angeles, CA 1999 (American Institute of Aeronautics and astronautics, Washington, DC, 1999).

[20] K. Makowski, Z. Peradzynski, N. Gascon and M. Dudeck, AIAA paper 99-2295, 35th Joint Propulsion Conference, Los Angeles, CA 1999 (American Institute of Aeronautics and astronautics, Washington, DC, 1999).

[21] A. Cohen-Zur, A. Fruchtman, and J. Ashkenazy, "Steady Acceleration in the Hall thruster", in $26^{\text {th }}$ International Electric Propulsion Conference, Kitakyushu, Japan, 1999. IEPC 99-108.

[22] V. Yu. Fedotov, A. A. Ivanov, G. Guerrini, A. N. Vesselovzorov, and M. Bacal, Phys. Plasmas 6, $4360(1999)$.

[23] M. Keidar and I. D. Boyd, J. Appl. Phys. 86, 4786 (1999). 
[24] V. V. Zhurin, H. R. Kaufman, and R. S. Robinson, Plasma Sources Sci. Technol. 8, R1 (1999).

[25] E. Y. Choueiri, "Fundamental differences between the stationary plasma thruster and the anode layer thruster", Bull. Am. Phys. Society 44, 61 (1999).

[26] Y. Raitses, L. A. Dorf, A. A. Litvak, and ,N. J. Fisch, J. Appl. Phys. 88, 1263 (2000).

[27] A. Fruchtman and N. J. Fisch, "Variational principle for optimal accelerated neutralized flow", submitted to Phys. Plasmas.

[28] K. Oswatitsch, “Gas Dynamics” (Academic, New York, 1956), Chap. 2.

[29] E. L. Resler, Jr., and W. R. Sears, Journal of the Aeronautical Sciences, p. 235 (1958).

[30] Ch. Wieckert, Phys. Fluids 30, 1810 (1987).

[31] E. N. Parker, “Interplanetary Dynamical Processes” (Interscience publishers, New York, 1963).

[32] K.-U. Riemann, J. Phys. D: Appl. Phys. 24, 493 (1991); Phys. Fluids B 3, 3331 (1991).

[33] M. Martinez-Sanchez, J. Propulsion and Power, 56 (1991).

[34] J. Ashkenazy, Physics Letters A 228, 369 (1997).

[35] D. Bohm, in "The characteristics of Electrical Discharges in Magnetic Fields", edited by A. Guthry and R. K. Wakerling (McGraw - Hill, New York, 1949), Chap. 3.

[36] R. E. Duvall, A. Fruchtman, Y. Maron, and L. Perelmutter, Phys. Fluids B5, 3399 (1993).

[37] N. Hershkowitz, Space Sci. Rev. 41, 351 (1985).

[38] S. I. Braginskii, “Transport processes in a plasma,"in Reviews of Plasma Physics, Vol. 1 (Consultants Bureau, New York, 1965), p. 205. 


\section{Figure Captions}

Fig. 1 Schematic drawing of the Hall thruster.

Fig. 2 High efficiency case: $p=1.42,1 / \mu_{0}=19, q=0, T_{e} / e \phi_{A}=0.05$. Shown are the axial profiles of the normalized ion current $(J)$, ion velocity $(V)$, plasma density $(N)$, electric potential $(\psi)$, electron current $\left(J_{e}\right)$, and total current $\left(J_{T}\right)$ along the channel. The dashed lines correspond to the regular Hall thruster and the solid lines to the Hall thruster with the additional electrode. The additional electrode is located at $\xi=0.3$.

Fig. 3 Low efficiency case. The parameters are: $p=0.75,1 / \mu_{0}=3, q=0, T_{e} / e \phi_{A}=0.05$. The variables are as in Fig. 2. The additional electrode is located at $\xi=0.4$.

Fig. 4 High temperature case. The parameters are: $p=1,1 / \mu_{0}=1 / 3, q=$ $0.1, T_{e} / e \phi_{A}=0.5$. The variables are as in Fig. 2. The additional electrode is located at $\xi=0.8$. 
M agnetic circuit

$\mathrm{C}$ athode neutralizer

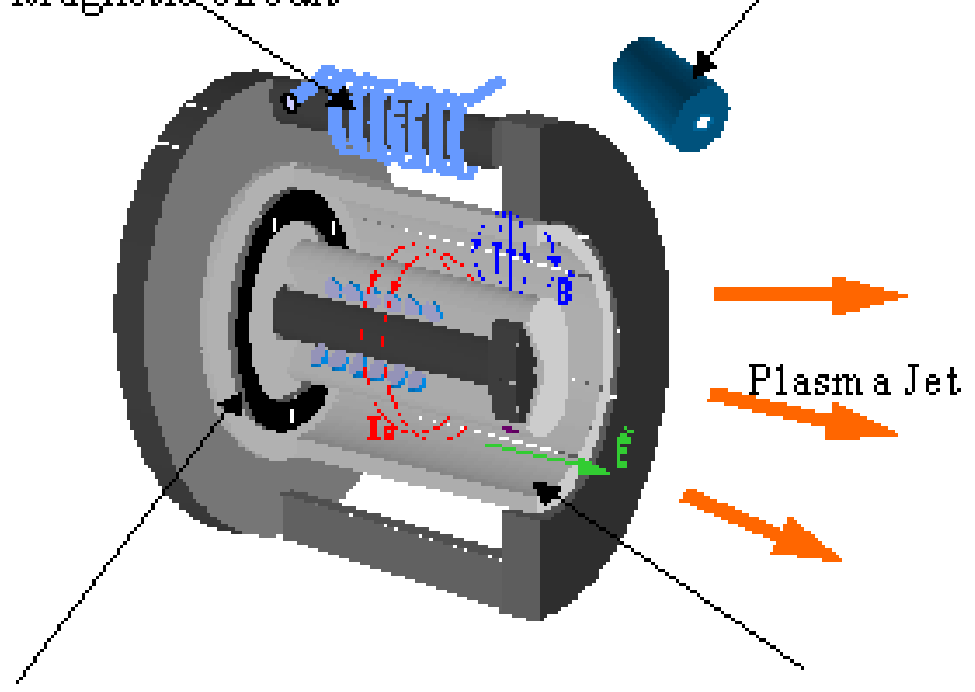

Anode/gas distributor

Insulator $\mathrm{C}$ hanne 1 

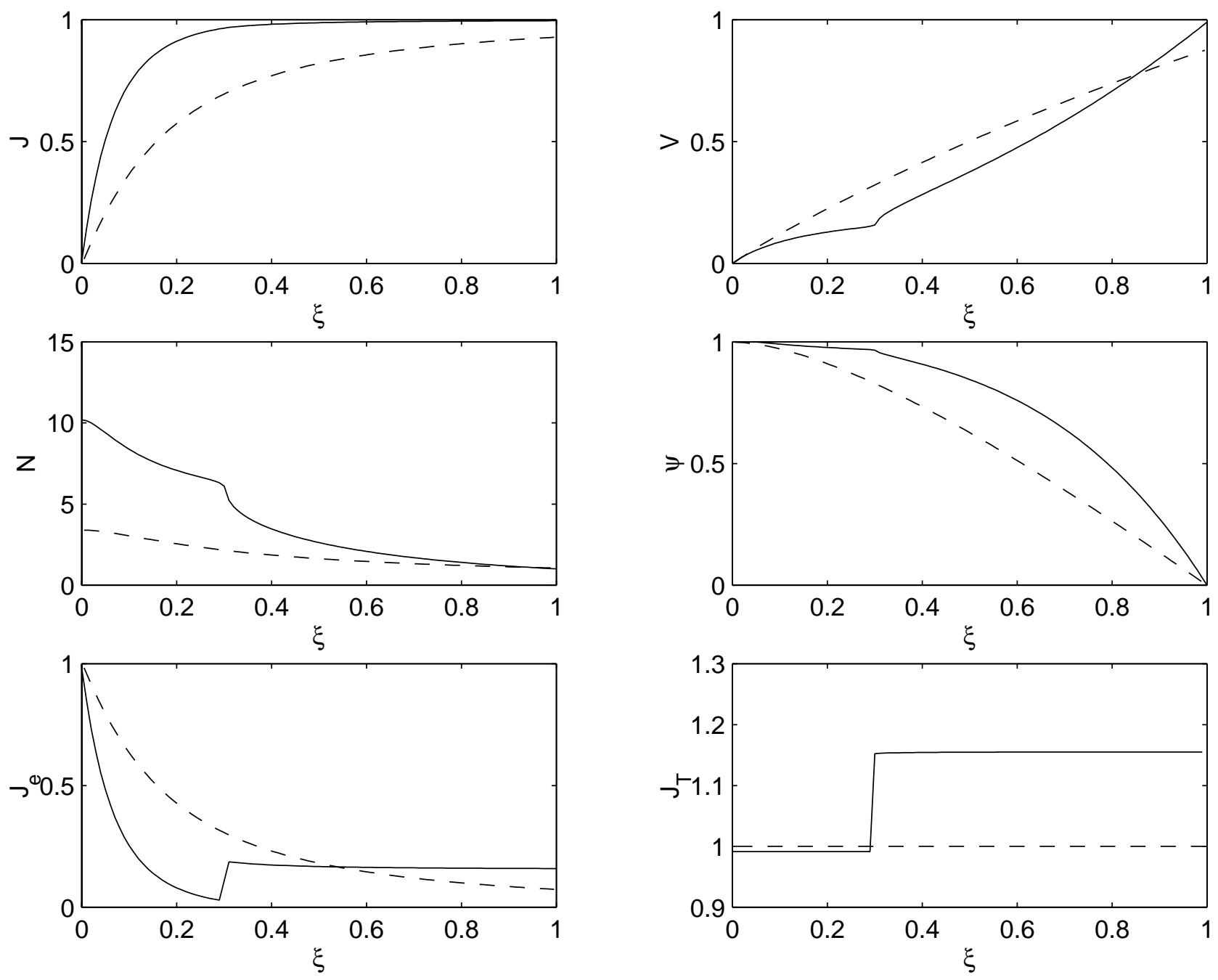

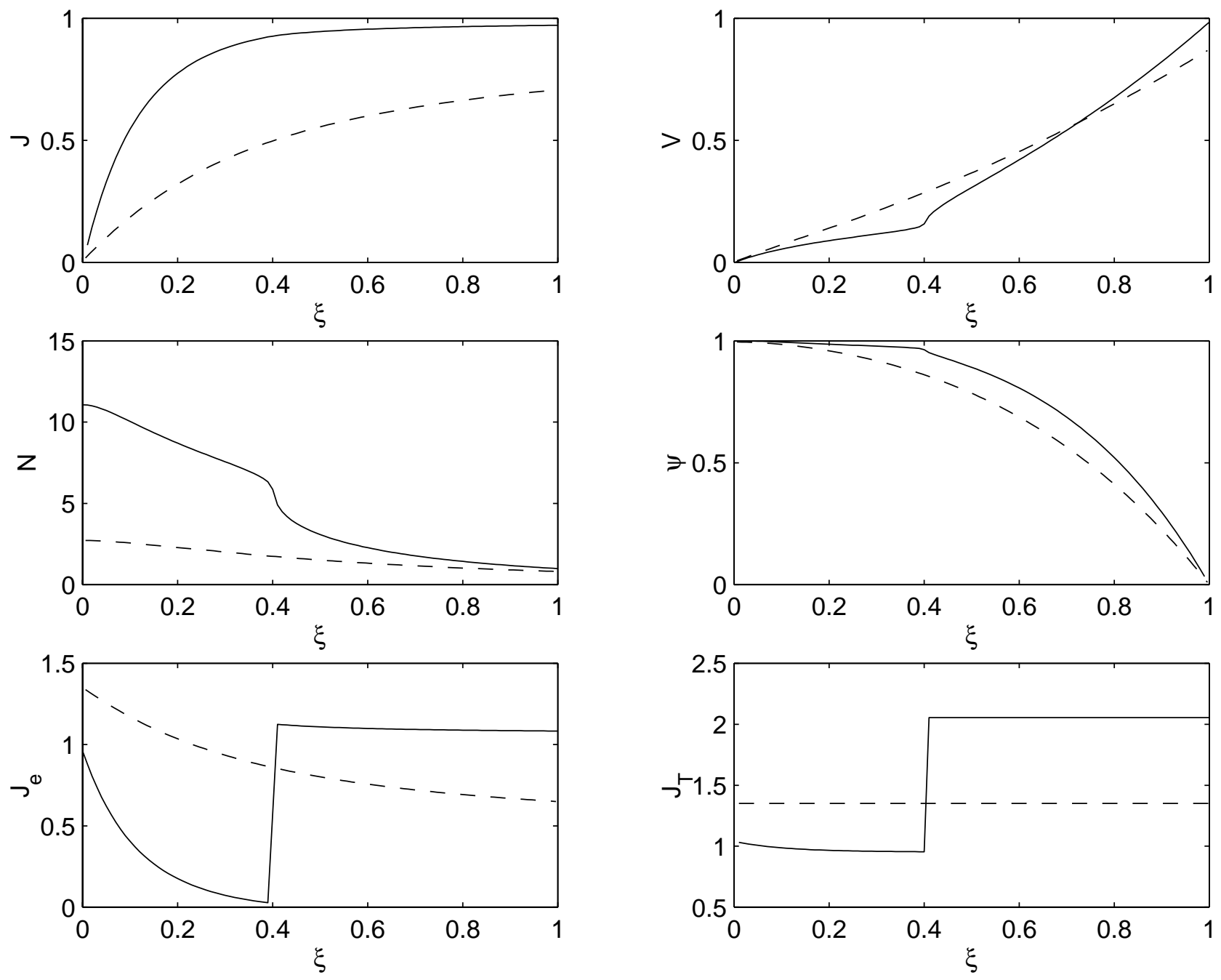

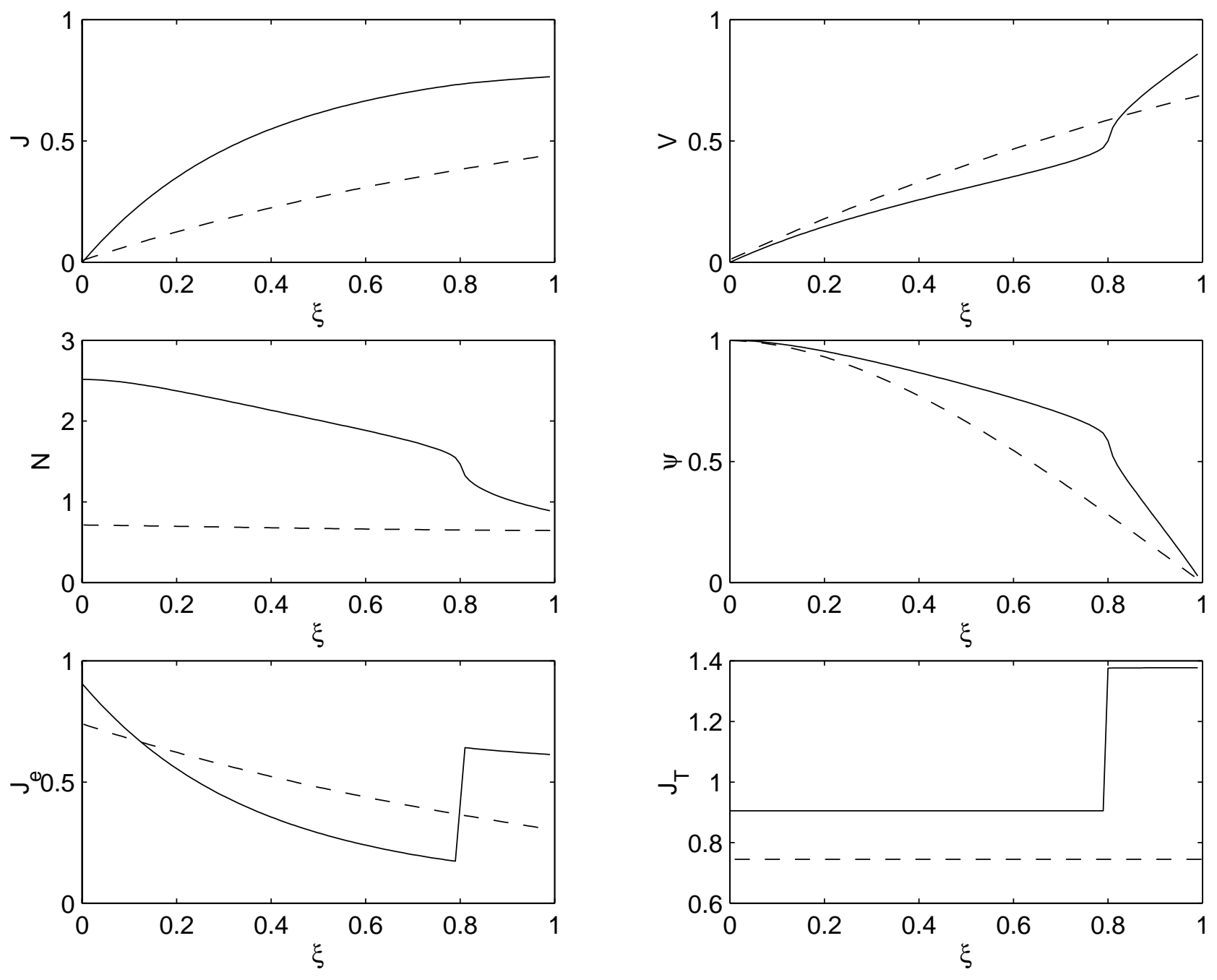
The Princeton Plasma Physics Laboratory is operated by Princeton University under contract with the U.S. Department of Energy.

\author{
Information Services \\ Princeton Plasma Physics Laboratory \\ P.O. Box 451 \\ Princeton, NJ 08543
}

Phone: 609-243-2750

Fax: 609-243-2751

e-mail: pppl_info@pppl.gov

Internet Address: http://www.pppl.gov 